\title{
SOLAR DYNAMICS OBSERVATORY DISCOVERS THIN HIGH TEMPERATURE STRANDS IN CORONAL ACTIVE REGIONS
}

\author{
Fabio Reale $^{1,2}$, Massimiliano Guarrasi $^{1}$, Paola Testa $^{3}$, Edward E. DeLuca $^{3}$, Giovanni Peres $^{1,2}$, and Leon Golub $^{3}$ \\ ${ }^{1}$ Dipartimento di Fisica, Università di Palermo, Piazza del Parlamento 1, 90134 Palermo, Italy \\ 2 INAF-Osservatorio Astronomico di Palermo "G. S. Vaiana," Piazza del Parlamento 1, 90134 Palermo, Italy \\ ${ }^{3}$ Harvard-Smithsonian Center for Astrophysics, Cambridge, MA 02138, USA \\ Received 2011 March 18; accepted 2011 June 8; published 2011 June 29
}

\begin{abstract}
One scenario proposed to explain the million degree solar corona is a finely stranded corona where each strand is heated by a rapid pulse. However, such fine structure has neither been resolved through direct imaging observations nor conclusively shown through indirect observations of extended superhot plasma. Recently, it has been shown that the observed difference in the appearance of cool and warm coronal loops $(\sim 1 \mathrm{MK}$ and $\sim 2-3$ MK, respectively)—warm loops appearing "fuzzier" than cool loops—can be explained by models of loops composed of subarcsecond strands, which are impulsively heated up to $\sim 10 \mathrm{MK}$. That work predicts that images of hot coronal loops ( $\gtrsim 6 \mathrm{MK}$ ) should again show fine structure. Here we show that the predicted effect is indeed widely observed in an active region with the Solar Dynamics Observatory, thus supporting a scenario where impulsive heating of fine loop strands plays an important role in powering the active corona.
\end{abstract}

Key words: Sun: corona - Sun: UV radiation

Online-only material: color figures

\section{INTRODUCTION}

The bright corona consists of magnetic loop-like tubes which confine the heated plasma. It has been proposed that the plasma is heated by rapid energy pulses, the so-called nanoflares, due to very localized reconnections of the magnetic field braided and twisted by the chaotic motions of the loop footpoints in the photosphere (Parker 1988; Cargill 1994; Cargill \& Klimchuk 2004). Although impulsive events were predicted to be small and rapid, they are expected to produce hot, variable emission at $\sim 10 \mathrm{MK}$, even in the absence of flares. The evidence for these signatures has been neither direct nor conclusive so far (Reale et al. 2009a, 2009b; McTiernan 2009; Schmelz et al. 2009; Sylwester et al. 2010). The lack of evidence suggests that alternative mechanisms, i.e., the more gradual dissipation of MHD Alfven waves (Hollweg 1984; Nakariakov et al. 1999; Ofman \& Wang 2008), or even the direct involvement of the underlying chromosphere (De Pontieu et al. 2011), are important (even critical) to the solution of the heating problem.

Indeed, there are good reasons why extensive nanoflaring activity has been elusive so far (Klimchuk 2006; Reale 2010). An important one is that, since momentum and energy are transported only along the magnetic field lines, a single pulse heats just one long and thin strand at a time. These strands are too thin to be spatially resolved by current telescopes, and the detection of hot plasma is difficult because of its small filling factor (Martens et al. 1985).

Recently, it has been shown (Guarrasi et al. 2010) that coronal loops substructured in a multitude of thin strands pulse-heated up to $10 \mathrm{MK}$ can explain the evidence of increasing fuzziness with temperature of emitting plasma (e.g., Brickhouse \& Schmelz 2006; Tripathi et al. 2009). This model also predicts that the fuzziness will decrease in bands sensitive to temperature $>3 \mathrm{MK}$. Here we show that this prediction is indeed confirmed by active region observations with the Solar Dynamics Observatory $(S D O)$.
We suppose that, in the multi-stranded loops that populate an active region, the duration of each energy pulse is much smaller than the plasma characteristic radiative and conductive cooling times (Serio et al. 1991). Once the heat pulse ends, the plasma cools down exponentially. If each loop strand is heated infrequently, i.e., the time between heating events is comparable or larger than the radiative cooling time, the plasma in the strand will be at high temperature only for a short time, and cooling for most of the time. Therefore, at a given time, only a few strands will be very hot, and many of them will be significantly cooler than their peak temperature. If nanoflares are scaled-down versions of coronal flares, we expect peak temperatures of about $10 \mathrm{MK}$. On the other hand, while the plasma is so hot, its emission is faint because of its low density: dense (and bright) plasma comes up from the chromosphere on timescales longer than the duration of the heat pulses. Therefore, the strands become bright when the plasma is already cooling and remain bright until the plasma has drained significantly. More specifically, it has been shown that a strand heated to $10 \mathrm{MK}$ for $\sim 1$ minute is at its brightest when it has cooled to $\sim 3 \mathrm{MK}$, and that it remains around this temperature for a relatively long time (Guarrasi et al. 2010). Thus, in this scenario, we expect to see few hot $10 \mathrm{MK}$ strands, and many $3 \mathrm{MK}$ strands in active regions.

\section{DATA ANALYSIS AND MODELING}

The $S D O$ was launched in 2010 February. The Atmospheric Imaging Assembly (AIA), with its seven EUV narrowband channels, images the solar corona with high spatial resolution $\left(\sim 0.6\right.$ arcsec pixel $\left.^{-1}\right)$, and high cadence in several spectral bands at the same time. We analyze $S D O$ /AIA observations of AR11117 on 2010 October 28, from 2:00 UT. We consider a $500 \times 500$ pixel region in three channels: $171 \AA$, $335 \AA$, $94 \AA$. The narrow passbands of the AIA channels contain bright spectral lines emitted by plasma at different temperatures. In 


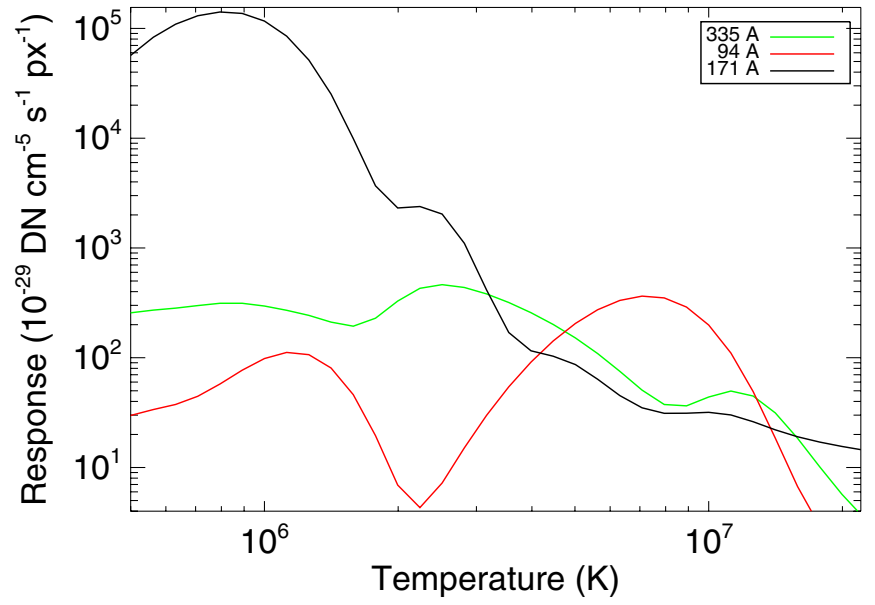

Figure 1. Instrument response per unit emission measure and as a function of temperature, for the $94 \AA$, $335 \AA$, and $171 \AA$ SDO/AIA channels.

(A color version of this figure is available in the online journal.)

particular, the channels centered at $94 \AA$ and $335 \AA$ contain a strong line of Fe XVIII and Fe XVI, respectively, which are emitted more efficiently by plasma around $8 \mathrm{MK}$ and $3 \mathrm{MK}$ (O'Dwyer et al. 2010). Therefore, these two channels are appropriate to test the prediction of the model.

We use level-1.0 data, after standard processing of level0 data (bad-pixel removal, despiking, flat fielding). Data are obtained from a standard observing series with cadence of $12 \mathrm{~s}$ in all channels, and exposure times of $2 \mathrm{~s}$ in the $171 \AA$ channel and $2.9 \mathrm{~s}$ in the $335 \AA$ and $94 \AA$ channels. The channels have very different sensitivity to the solar coronal emission (Figure 1), resulting in different signal-to-noise ratios. Therefore, for a more meaningful comparison of the active region morphology in the different channels, and to avoid possible spurious effects due to the different noise level, we summed images in the lower intensity channels: first we co-aligned the images in each channel by using a standard cross-correlation routine (tr_get_disp.pro in IDL SolarSoftware package), and then we added 30 consecutive images in the $94 \AA$ channel and three in the $335 \AA$ channel (noise of $\sim 3 \%-4 \%$ in both channels in the central region of Figure 2). Final images in all three channels were then co-aligned again. We note that during the $94 \AA$ time interval ( $\approx 7$ minutes) the region variability is of a few percent at most, and that our conclusions on the observed morphological differences in the hotter versus cooler emission are, if anything, underestimated by the temporal averaging we effectively apply by summing up images.

Figure 2 (top three images) shows the internal part of the active region in the $94 \AA, 335 \AA$, and $171 \AA$ channels. The $171 \AA$ channel contains a very strong Fe IX line with peak emissivity at $\approx 0.8 \mathrm{MK}$. For the moment, we focus our attention to the top two images and, in particular, to the core of the active region.

In the $335 \AA$ channel, the active region core is covered quite uniformly by a large number of bright arches. In the southern part (20 arcsec $\lesssim Y \lesssim 55 \operatorname{arcsec}$ ), the arches coalesce to form a uniform bright band. In the northern part, we can identify three brighter loop groups in a background of more diffuse emission. A few very bright spots are visible at the center of the region. Overall the region has quite a diffuse appearance and individual loops cannot be clearly resolved.

In the $94 \AA$ channel, overall we see a very similar morphology and many bright structures are clearly cospatial with those observed in the $335 \AA$ channel. The most striking difference
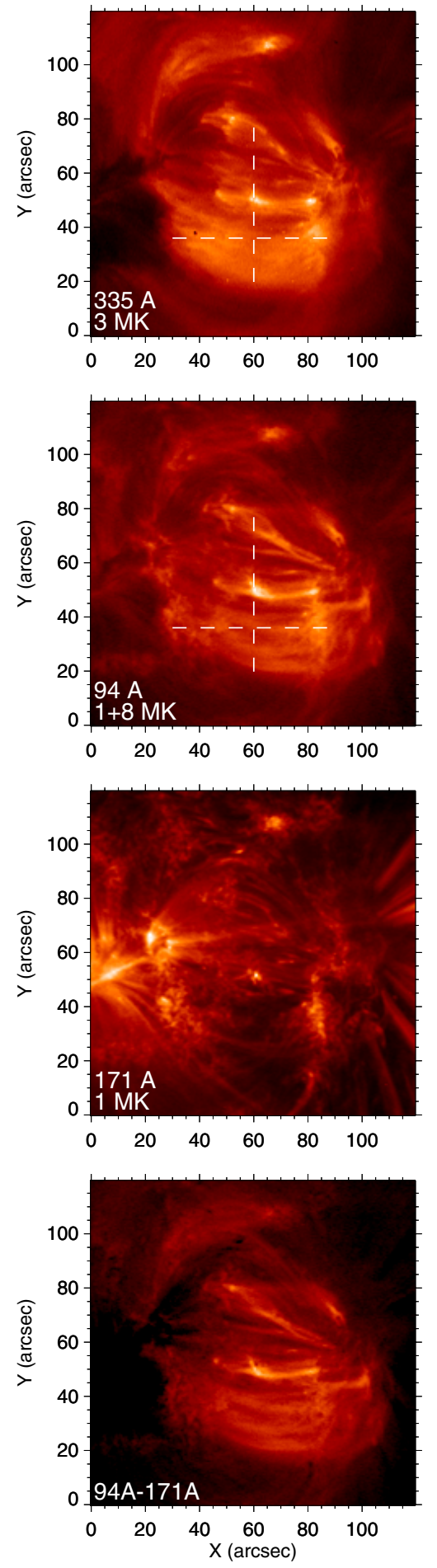

Figure 2. Inner part of the active region AR11117 observed in three different channels ( $335 \AA$, $94 \AA$, $171 \AA$ ) of the SDO/AIA on 2010 October 27 around 02 UT. The color scales as the square root of the pixel counts. The ranges are 59-1567, 55-3157, 155-4681 DN for the three channels, respectively. The channels are most sensitive to plasma emitting at the labeled temperatures. The bottom panel shows the image obtained by subtracting the cool component scaled from the $171 \AA$ channel to the $94 \AA$ image (same color scale as second panel, real range 0-2741 DN). Figure 3 shows brightness profiles along the marked vertical and horizontal lines.

(A color version of this figure is available in the online journal.)

from the image in the other channel is in the core itself: in the southern part, while in $335 \AA$ the arches are densely packed and uniform, in $94 \AA$ they have greater contrast, i.e., we see an 
alternation of bright and fainter structures. In the northern part, we are even able to resolve very thin bright east-to-west bridges, in the same location where thicker arches are present in the softer channel. Overall, in the $94 \AA$ channel, the loop systems appear sharper, the observed emission largely less "fuzzy," and we can resolve thinner bright structures than in the $335 \AA$ channel. This is exactly the effect that we expected, and that was predicted (Guarrasi et al. 2010).

We have to be cautious, however, in one important point. Although the channel passbands are narrow, they include several spectral lines. In particular, the $94 \AA$ channel includes another strong line $(\mathrm{Fe} \mathrm{x}$ ) which peaks at $\sim 1 \mathrm{MK}$. In general, we cannot be sure that the emission imaged by this channel comes only from hot plasma ( $\gtrsim 6 \mathrm{MK}$ ). The $171 \AA$ image helps us in this respect, because it allows us to localize the bright cooler plasma ( $T \sim 1 \mathrm{MK}$ ), and to assess whether the $94 \AA$ emission is due to hot or cool plasma. In the $171 \AA$ channel, the active region shows quite a different morphology. Many structures are complementary to those observed in the other channels (Reale et al. 2007). The core appears depleted of arch-like structures. Only a few of them are visible, and they look quite different from those in both the other channels. The arch-like structures are instead replaced by bright "moss." This moss is a well-known feature of this soft channel, already studied in Normal Incidence X-ray Telescope (NIXT) and Transition Region and Coronal Explorer (TRACE) observations and commonly explained as the bright warm footpoints of (hot) high-pressure loops (Peres et al. 1994; Fletcher \& de Pontieu 1999; Martens et al. 2000). The $171 \AA$ image clearly indicates that much of the plasma confined in the filamented arches that we see in the $94 \AA$ channel is not warm at $1 \mathrm{MK}$, and therefore it must be hot around 6-8 MK. As a further test, we estimated the emission measure of the cool plasma from the $171 \AA$ map and used it to compute the expected contribution of the cool plasma in the $94 \AA$ channel. We made the conservative assumptions that the cool plasma temperature is the one of the peak of the cool $94 \AA$ response component (Figure 1), i.e., $\log T=6.05$, and that the cool $94 \AA$ response component is underestimated by a factor five (Aschwanden \& Boerner 2011). We subtracted this estimated cool $94 \AA$ map from the $94 \AA$ map shown in Figure 2. The resulting image is also shown in Figure 2 (bottom). We note that the moss emission visible in the $94 \AA$ channel is considerably reduced, and that the loop footpoints regions and the outbound loops are mostly removed. On the other hand, the emission of the loop bundle in the central region does not change significantly, except for a reduction in the moss region, and the fine structures in the upper half of the image are also almost unchanged. This confirms that the emission of the loop bundle and of the fine structures mostly comes from hot plasma.

For a quantitative estimate of the different fuzziness of the hot ( $\gtrsim 6 \mathrm{MK})$ and cooler ( $~ 3 \mathrm{MK}$ ) plasma, Figures 3(a) and (b) plot the pixel brightness, divided by a moving average, along the vertical and horizontal lines in Figure 2. The brightness of the $94 \AA$ channel after subtracting the cool $1 \mathrm{MK}$ component is also shown. We note that all the steep gradients are traced by more than one data point, i.e., the trends are coherent and are due to real structures not to noise. Along the horizontal line - that runs approximately along the magnetic tubes-the pixel brightness changes in a similar way and with a similar amplitude in both channels. Table 1 shows the root-meansquare (rms) averages of the amplitude excursion, i.e., $\approx 3 \%$ and $\approx 5 \%-6 \%$, respectively. Along the vertical line-which runs across the field line direction-both brightnesses in the $94 \AA$ channel are significantly more variable in space than in the $335 \AA$ channel, and the rms average of the amplitude excursion becomes $10 \%-13 \%$ versus $6 \%$.

To check on larger baselines, we derived the analogous values over all the rows $(X)$ and columns $(Y)$ of selected regions. For each region, Table 1 shows the rms average values, and their standard deviations, in both channels. We selected three regions: one with as little moss as possible, the very center, and a larger core region. For the second region, we also report the values obtained in the $94 \AA$ channel after subtracting the cool component. We find that the $Y$-values in the $94 \AA$ channel are invariably much higher than all the others, thus confirming that the decrease of fuzziness in the $94 \AA$ channel is highly significant.

To add support to this evidence, we take the two-dimensional fast Fourier transforms (FFTs) of the images of Figure 2 in the $94 \AA$ channel before and after subtracting the cool component and in the $335 \AA$ channel. Then we sum along circles of constant wavenumber, as it was done in the past for NIXT observations (Gomez et al. 1993a, 1993b). The power at zero wavenumber reflects the mean intensity of the image, so we normalize the images by the average intensity to make cross comparison of the power distributions more straightforward. In Figure 3(c), the resulting Fourier transforms are power laws. We see that both $94 \AA$ FFTs are systematically higher than the $335 \AA$ FFT in the wavenumber range $\sim 10-70$ that corresponds to a spatial range $\sim 14-2$ pixels. This range is in agreement with the crosssection size of the observed structures. All the lines converge at the highest wavenumbers, which are related to the presence of noise. This indicates a similar signal-to-noise ratio for both channels. In the end, the figure confirms more power at relatively high wavenumbers in the $94 \AA$ channel both with and without subtracting the $1 \mathrm{MK}$ component.

For reference, we now show some results of hydrodynamic modeling of loops consisting of thin pulse-heated strands (Guarrasi et al. 2010). Each pulse lasts $60 \mathrm{~s}$ and brings the plasma temporarily to $\sim 10 \mathrm{MK}$. The plasma then cools off freely. Images comparable to the observed ones are obtained, for instance, by assuming that an instrument resolution element includes about 60 unresolved strands. Each strand is symmetric with respect to the loop apex. Figure 4 shows the strandedloop emission obtained by folding the density and temperature profiles from the hydrodynamic model with the $94 \AA$ and $335 \AA$ channels responses. Only the coronal part of the loop (upper $75 \%$ of the loop length) is shown. To emphasize the effect we are addressing, we normalize the emission in each pixel strip across the loop. In Figure 4 we can clearly see more white and black thin strips in the $94 \AA$ image, while the other is more uniformly colored.

The rms average of the amplitude excursion obtained from this model is $17 \%$ for $94 \AA$ channel, and $9 \%$ for $335 \AA$ channel, not too far from those measured in the data. As explained by Guarrasi et al. (2010), the ultimate reason for this behavior is that overall each strand spends proportionally a long time, and with a high emission measure, at temperatures around $3 \mathrm{MK}$, i.e., just the temperature of maximum sensitivity of the $335 \AA$ channel. Extrapolating this result to a whole active region, threaded with thousands of magnetic field lines, the loops will therefore be more populated by bright strands and appear more uniformly bright in this channel. On the contrary, they will appear less "filled" in a hotter channel, consistently with what we observe in the $94 \AA$ channel. 


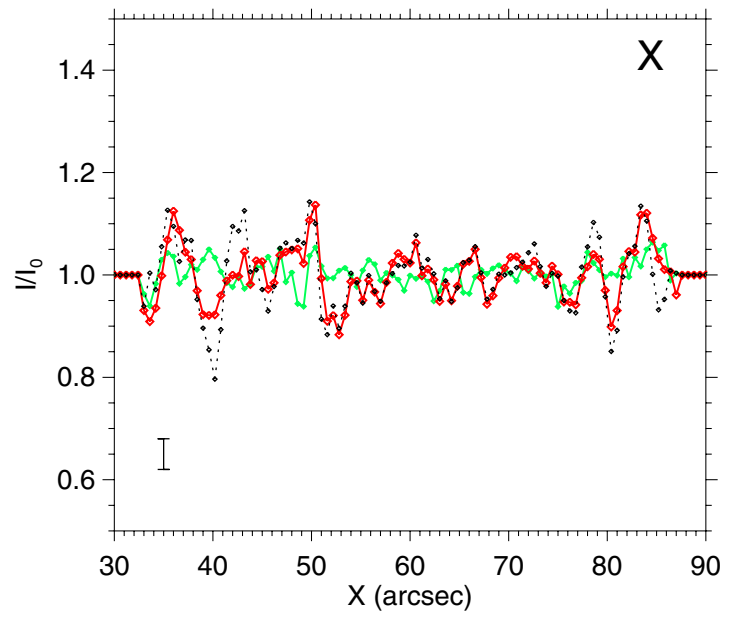

(a)

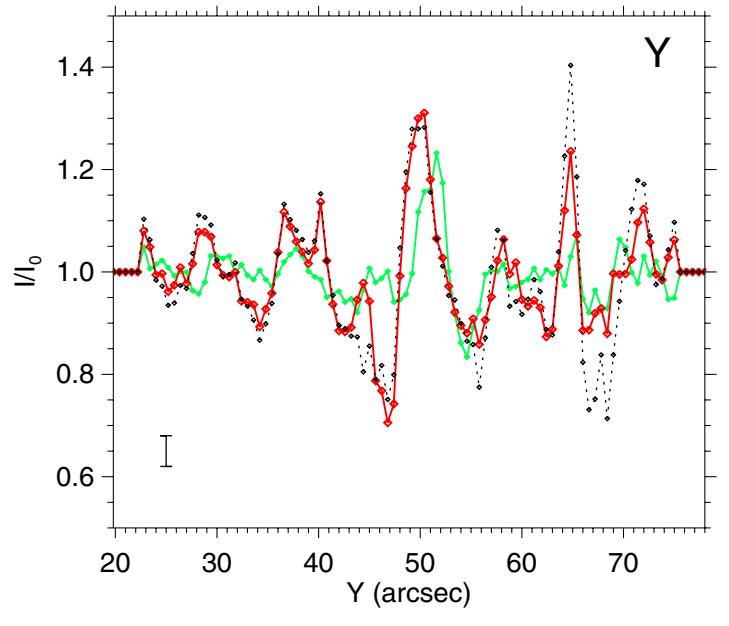

(b)

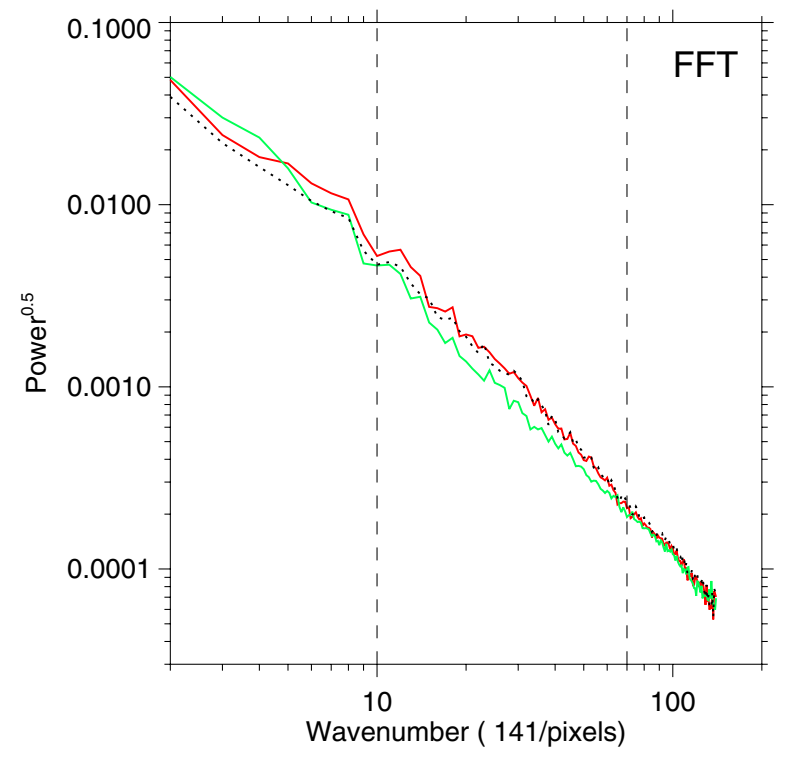

(c)

Figure 3. Brightness profiles in the $94 \AA$ before (red) and after (black dotted) subtracting the cool 1 MK component, and in the $335 \AA$ (green) channels along the horizontal (a) and vertical (b) lines in Figure 2. The profiles are normalized to a moving average with a 10 pixel boxcar. A typical error bar is also shown. (c) Normalized two-dimensional Fourier transforms of $335 \AA$ and both $94 \AA$ images in Figure $2.94 \AA$ transforms are both systematically higher than the $335 \AA$ transform in the wavenumber range between the dashed lines.

(A color version of this figure is available in the online journal.)

Table 1

Fractional rms Amplitude Excursions

\begin{tabular}{lcccccccc}
\hline \hline Region & $335 \AA X$ & $\sigma$ & $94 \AA X$ & $\sigma$ & $335 \AA Y$ & $\sigma$ & $94 \AA Y$ & $\sigma$ \\
\hline Single row/column & 0.03 & $\ldots$ & 0.05 & $\ldots$ & 0.06 & $\ldots$ & 0.10 & $\ldots$ \\
Region little moss $($ size: $60 \times 60$ pixels, & 0.032 & 0.017 & 0.046 & 0.021 & 0.052 & 0.011 & 0.082 & 0.017 \\
center: $\left.\left[X=58^{\prime \prime}, Y=38^{\prime \prime}\right]\right)$ & & & & & & & & \\
Central core region $\left(70 \times 70,\left[68^{\prime \prime}, 49^{\prime \prime}\right]\right)$ & 0.044 & 0.018 & 0.059 & 0.021 & 0.072 & 0.016 & 0.111 & 0.036 \\
Central core region $(94-171 \AA)$ & 0.044 & 0.018 & 0.068 & 0.025 & 0.072 & 0.016 & 0.130 & 0.037 \\
Whole core region $\left(100 \times 100,\left[72^{\prime \prime}, 48^{\prime \prime}\right]\right)$ & 0.053 & 0.021 & 0.065 & 0.021 & 0.063 & 0.011 & 0.099 & 0.020 \\
\hline
\end{tabular}

Notes. The first row is computed along the lines marked in Figure 2. $\sigma$ is the standard deviation of the rms excursions.

Similar results are obtained with pulses that heat the plasma to slightly higher or lower temperatures $(\approx 20 \%-30 \%)$. Simulations and models no longer match the observations for weaker pulses leading to plasma maximum temperature $\sim 4 \mathrm{MK}$ and lower, where the cool $\sim 1 \mathrm{MK}$ component would dominate the observed emission in the $94 \AA$ channel. We can also exclude models with $10 \mathrm{MK}$ heat pulses lasting significantly less than 1 minute: the main hot emitting ions would not have the time to 


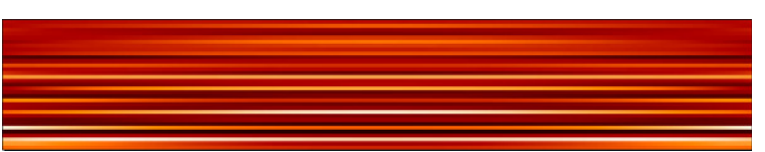

(a)

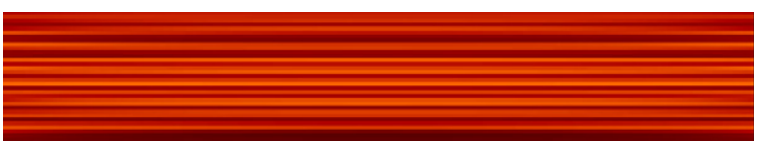

(b)

Figure 4. Emission of a (straightened) pulse-heated multi-stranded loop synthesized from hydrodynamic modeling in the (a) $94 \AA$ and (b) $335 \AA$ channels. The emission in each pixel strip is normalized across the loop. The color scale is linear between 0.6 and 1.4 (average 1): white is the maximum of emission, and black the minimum. Only the coronal part of the loop (upper $75 \%$ of the loop length) is shown.

(A color version of this figure is available in the online journal.)

reach ionization equilibrium (Reale \& Orlando 2008), therefore effectively suppressing the high-temperature emission, visible in the $94 \AA$ channel. As a warning, we remark that this model is tailored to describe a system of some loops, rather than an entire active region, and that the center of an active region may require a different (larger) number of strands per pixel.

As a further support, we only mention here that the model predicts the emission in the $94 \AA$ channel to vary in time with larger amplitude than in the $335 \AA$ channel, and that this is confirmed by the observation: the pixel light curves obtained from the model are in good agreement with the observed ones. We defer a detailed report on time variability to forthcoming work.

\section{CONCLUSIONS}

The new SDO active region data show evidence of extensive hot plasma, which is very finely structured, as expected in a scenario where storms of intense and rapid energy pulses are heating multi-stranded coronal loops. The strands are temporarily heated to flare temperatures. Our analysis is straightforward and little depends on processing details, such as background subtraction. We remark that in the $94 \AA$ channel we are not resolving the individual strands. We instead still see bundles of strands and the brightest ones are those where the fraction of very hot strands is relatively large. The reason why these very hot components are so difficult to detect is their small emission measure, and their small duty cycle with respect to most of their evolution time, spent mostly in the subsequent long cooling phase. This result supports previously debated analyses (Reale et al. 2009a, 2009b; Schmelz et al. 2009; McTiernan 2009; Sylwester et al. 2010), is consistent with many other pieces of evidence pointing to dynamically heated loops, e.g., overdensity of $\sim 1$ MK loops (Klimchuk 2006; Reale 2010), and supports localized heat pulses, e.g., nanoflares, playing an important role in powering coronal active regions.

F.R., G.P., and M.G. acknowledge support from Italian Ministero dell'Università e Ricerca and Agenzia Spaziale Italiana (ASI), contract I/023/09/0. P.T. was supported by contract SP02H1701R from Lockheed-Martin to the Smithsonian Astrophysical Observatory.

\section{REFERENCES}

Aschwanden, M. J., \& Boerner, P. 2011, ApJ, 732, 81

Brickhouse, N. S., \& Schmelz, J. T. 2006, ApJ, 636, L53

Cargill, P. J. 1994, ApJ, 422, 381

Cargill, P. J., \& Klimchuk, J. A. 2004, ApJ, 605, 911

De Pontieu, B., et al. 2011, Science, 331, 55

Fletcher, L., \& de Pontieu, B. 1999, ApJ, 520, L135

Gomez, D. O., Martens, P. C. H., \& Golub, L. 1993a, ApJ, 405, 767

Gomez, D. O., Martens, P. C. H., \& Golub, L. 1993b, ApJ, 405, 773

Guarrasi, M., Reale, F., \& Peres, G. 2010, ApJ, 719, 576

Hollweg, J. 1984, ApJ, 277, 392

Klimchuk, J. A. 2006, Sol. Phys., 234, 41

Martens, P. C. H., Kankelborg, C. C., \& Berger, T. E. 2000, ApJ, 537, 471

Martens, P. C. H., van den Oord, G. H. J., \& Hoyng, P. 1985, Sol. Phys., 96, 253 McTiernan, J. M. 2009, ApJ, 697, 94

Nakariakov, V., Ofman, L., DeLuca, E., Roberts, B., \& Davila, J. 1999, Science, 285,862

O’Dwyer, B., Del Zanna, G., Mason, H. E., Weber, M. A., \& Tripathi, D. 2010, A\&A, 521, A21

Ofman, L., \& Wang, T. 2008, A\&A, 482, L9

Parker, E. N. 1988, ApJ, 330, 474

Peres, G., Reale, F., \& Golub, L. 1994, ApJ, 422, 412

Reale, F. 2010, Living Rev. Sol. Phys., 7, 5

Reale, F., McTiernan, J. M., \& Testa, P. 2009a, ApJ, 704, L58

Reale, F., \& Orlando, S. 2008, ApJ, 684, 715

Reale, F., Testa, P., Klimchuk, J. A., \& Parenti, S. 2009b, ApJ, 698, 756

Reale, F., et al. 2007, Science, 318, 1582

Schmelz, J. T., et al. 2009, ApJ, 704, 863

Serio, S., Reale, F., Jakimiec, J., Sylwester, B., \& Sylwester, J. 1991, A\&A, 241, 197

Sylwester, B., Sylwester, J., \& Phillips, K. J. H. 2010, A\&A, 514, A82

Tripathi, D., Mason, H. E., Dwivedi, B. N., del Zanna, G., \& Young, P. R. 2009, ApJ, 694, 1256 